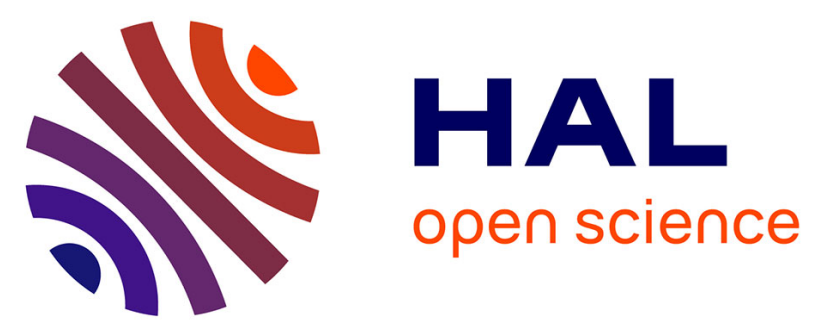

\title{
Extended-spectrum $\beta$-lactamase-producing in Spain belong to a large variety of multilocus sequence typing types, including ST10 complex/A, ST23 complex/A and ST131/B2
}

Jesús Oteo, Karol Diestra, Carlos Juan, Verónica Bautista, Ângela Novais, María Pérez-Vázquez, Bartolome Moyá, Elisenda Miró, Teresa M. Coque, Antonio Oliver, et al.

\section{- To cite this version:}

Jesús Oteo, Karol Diestra, Carlos Juan, Verónica Bautista, Ângela Novais, et al.. Extended-spectrum $\beta$-lactamase-producing in Spain belong to a large variety of multilocus sequence typing types, including ST10 complex/A, ST23 complex/A and ST131/B2. International Journal of Antimicrobial Agents, 2009, 34 (2), pp.173. 10.1016/j.ijantimicag.2009.03.006 . hal-00556336

\section{HAL Id: hal-00556336 https://hal.science/hal-00556336}

Submitted on 16 Jan 2011

HAL is a multi-disciplinary open access archive for the deposit and dissemination of scientific research documents, whether they are published or not. The documents may come from teaching and research institutions in France or abroad, or from public or private research centers.
L'archive ouverte pluridisciplinaire HAL, est destinée au dépôt et à la diffusion de documents scientifiques de niveau recherche, publiés ou non, émanant des établissements d'enseignement et de recherche français ou étrangers, des laboratoires publics ou privés. 


\section{Accepted Manuscript}

Title: Extended-spectrum $\beta$-lactamase-producing Escherichia coli in Spain belong to a large variety of multilocus sequence typing types, including ST10 complex/A, ST23 complex/A and ST131/B2

Authors: Jesús Oteo, Karol Diestra, Carlos Juan, Verónica

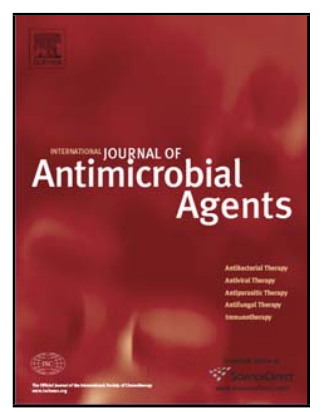

Bautista, Ângela Novais, María Pérez-Vázquez, Bartolome

Moyá, Elisenda Miró, Teresa M. Coque, Antonio Oliver, Rafael Cantón, Ferran Navarro, José Campos

PII:

S0924-8579(09)00117-4

DOI: doi:10.1016/j.ijantimicag.2009.03.006

Reference: ANTAGE 3010

To appear in: International Journal of Antimicrobial Agents

Received date: 26-1-2009

Revised date: 27-2-2009

Accepted date: $\quad 3-3-2009$

Please cite this article as: Oteo J, Diestra K, Juan C, Bautista V, Novais Â, PérezVázquez M, Moyá B, Miró E, Coque TM, Oliver A, Cantón R, Navarro F, Campos $\mathrm{J}$, Extended-spectrum $\beta$-lactamase-producing Escherichia coli in Spain belong to a large variety of multilocus sequence typing types, including ST10 complex/A, ST23 complex/A and ST131/B2, International Journal of Antimicrobial Agents (2008), doi:10.1016/j.ijantimicag.2009.03.006

This is a PDF file of an unedited manuscript that has been accepted for publication. As a service to our customers we are providing this early version of the manuscript. The manuscript will undergo copyediting, typesetting, and review of the resulting proof before it is published in its final form. Please note that during the production process errors may be discovered which could affect the content, and all legal disclaimers that apply to the journal pertain. 


\section{Extended-spectrum $\beta$-lactamase-producing Escherichia coli in} Spain belong to a large variety of multilocus sequence typing types, including ST10 complex/A, ST23 complex/A and

\section{ST131/B2}

Jesús Oteo ${ }^{a}$, Karol Diestra ${ }^{b}$, Carlos Juan ${ }^{c}$, Verónica Bautista ${ }^{a}$, Ângela Novais ${ }^{d}$, María Pérez-Vázquez ${ }^{a}$, Bartolome Moyá ${ }^{c}$, Elisenda Miró ${ }^{b}$, Teresa M. Coque

${ }^{d}$, Antonio Oliver ${ }^{c}$, Rafael Cantón ${ }^{d}$, Ferran Navarro ${ }^{b}$, José Campos ${ }^{a, e, *}$; Spanish Network in Infectious Pathology Project (REIPI)

a Laboratorio de Antibióticos, Servicio de Bacteriología, Centro Nacional de Microbiología, Instituto de Salud Carlos III, Madrid, Spain

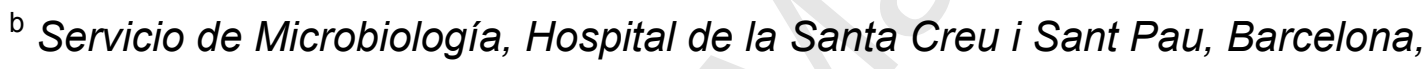
Spain

c Servicio de Microbiología, Hospital Son Dureta, Palma de Mallorca, Spain

d Servicio de Microbiología, Hospital Universitario Ramón y Cajal, CIBER en Epidemiología y Salud Pública (CIBERESP) and Unidad de Resistencia a Antibióticos y Virulencia Bacteriana Asociada al Consejo Superior de Investigaciones Científicas (CSIC), Madrid, Spain

e Consejo Superior de Investigaciones Científicas, Madrid, Spain

Received 26 January 2009; accepted 3 March 2009

Keywords: ESBL; MLST; Escherichia coli; Phylogenetic group 
* Corresponding author. Present address: Centro Nacional de Microbiología, Instituto de Salud Carlos III, Carretera Pozuelo a Majadahonda, 28220 Majadahonda, Madrid, Spain.

E-mail address: jcampos@isciii.es (J. Campos). 


\section{Abstract}

In this study, we investigated the population structure of extended-spectrum $\beta$ lactamase (ESBL)-producing Escherichia coli in Spain and determined possible associations between specific multilocus sequence typing (MLST) types and ESBL types. Ninety-two ESBL-producing E. coli isolates from 11 Spanish hospitals were studied. The predominant ESBLs in this collection were CTX-M14 (45.7\%), SHV-12 (21.7\%) and CTX-M-9 (20.6\%). Phylogenetic groups and MLST types were studied. Thirty-seven isolates (40.2\%) belonged to phylogroup A, 26 (28.3\%) to group B1, 13 (14.1\%) to group B2 and 16 (17.4\%) to group D. Fifty-six sequence types (STs) were identified, but ST131 (eight isolates) and ST167 (five isolates) were the most prevalent. The most common ST complexes were ST10 (13 isolates; $14.3 \%$ ) and ST23 (10 isolates; $11 \%$ ). Escherichia coli ST131 carried six different ESBLs (CTX-M-1, CTX-M-9, CTXM-10, CTX-M-14, CTX-M-15 and SHV-12), E. coli ST10 complex carried five ESBLs and E. coli ST23 complex carried four ESBLs. A great diversity of MLST types was observed among Spanish ESBL-producing E. coli isolates. 


\section{Introduction}

Most European countries have experienced a recent rapid dissemination of extended-spectrum $\beta$-lactamase (ESBL)-producing Escherichia coli isolates, in particular CTX-M-type $[1,2]$. In Spanish invasive E. coli isolates, ESBL production increased from 1.6\% in 2001 to $7 \%$ in 2007 (European Antimicrobial Resistance Surveillance System; http://www.rivm.nl/earss/). Although the spread of ESBLs is frequently due to the dissemination of mobile genetic elements [2-4], clonal dissemination of CTX-M-15-producing E. coli belonging to phylogenetic group B2 and ST131 has been addressed in different countries [5-8]. In addition, clonal spread of the CTX-M-9-group-producing ST38 strain has been described in a recent study in Japan [9]. The various methods by which ESBLs spread greatly influence the prevalence of different enzymes in specific areas.

Multilocus sequence typing (MLST) is expensive and time consuming, and almost all studies that characterise the population structure of ESBL-producing E. coli have selected the study population by some other criteria, such as serotyping [9] or production of specific ESBLs, e.g. CTX-M-15 [5,8]. As this approach may produce biased results, we sought to investigate the population genetics of $E$. coli carrying a variety of ESBLs without any previous selection.

Our study aimed to determine (i) the population structure of ESBL-producing $E$. coli in Spain and (ii) the possible association between specific MLST types and ESBL types. To attain these objectives, the population structure of a large, well 
characterised collection of 92 ESBL-producing $E$. coli isolates collected during a Spanish survey [10] was characterised.

\section{Material and methods}

A collection of 92 ESBL-producing $E$. coli isolates was recovered from clinical samples (68.5\% from urine) from 11 Spanish hospitals in five geographic regions (Andalucia, Cataluña, Madrid, Baleares and Valencia). All hospitals were members of the Spanish Network for Research in Infectious Diseases (REIPI) [10]. Participant hospitals were asked to collect up to 15 consecutive,

non-duplicate (one isolate per patient) ESBL-producing $E$. coli isolates by the first quarter of 2004. Eight different ESBL types were identified, with the predominant types being CTX-M-14 (45.7\%), SHV-12 (21.7\%) and CTX-M-9 (20.6\%), as described previously [10]. In addition, the plasmids encoding $b / a_{\mathrm{ESBL}}$ and the corresponding surrounding genes in selected isolates were further characterised [11].

In the present study, MLST types and phylogenetic groups of the ESBLproducing E. coli were analysed. Phylogenetic groups were determined by a multiplex polymerase chain reaction (PCR) assay as described by Clermont et al. [12]. Analysis of two genes (chuA and yjaA) and an anonymous DNA fragment (TSPE4.C2) allowed the identification of the four major phylogenetic groups of E. coli.

MLST determinations were carried out according to the Max-Planck Institute scheme for E. coli developed by M. Achtman et al. (http://web.mpiib- 
berlin.mpg.de). The phylogenetic relationships among the different sequence types (STs) found in this study were established according to the eBURST program version 2 (http://linux.mlst.net/burst.htm). STs of all E. coli isolates from the MLST database as well as STs of ESBL-producing E. coli from this study were considered together and introduced into the eBURST for analysis and elaboration of a population snapshot (Fig. 1).

Statistical analyses were performed using GraphPad Prism version 3.02 software (GraphPad Software Inc., San Diego, CA).

\section{Results}

\subsection{Phylogenetic groups}

Of the 92 ESBL-producing E. coli isolates, 37 (40.2\%) belonged to phylogenetic group A, $26(28.3 \%)$ to group B1, $13(14.1 \%)$ to group B2 and $16(17.4 \%)$ to group D. Seven different ESBL types were found among E. coli phylogroup A isolates, four among phylogroup B1 isolates, six among phylogroup B2 isolates and three among phylogroup $D$ isolates.

Escherichia coli producing the three most prevalent ESBL types (CTX-M-14, CTX-M-9 and SHV-12) were distributed among all four phylogroups, with CTXM-14-producing isolates being the most prevalent in all of them. Of the three CTX-M-15-producing isolates, two were phylogroup B2 and one was phylogroup A. 


\subsection{MLST analysis}

Among the 91 isolates tested ( 1 isolate could not be sequenced), 56 different STs were identified. The most prevalent STs were ST131 (eight isolates), ST167 (five isolates), ST23 (four isolates), ST602 (four isolates) and ST359 (four isolates) (Fig. 1; Table 1). The ST131 isolates were recovered from different geographic locations: Barcelona (four isolates from three different hospitals); Madrid (three isolates from one hospital); and Seville (one isolate).

By eBURST, 33 STs were distributed into 15 different ST complexes (53 isolates), whilst 23 STs were considered singletons (38 isolates) (Table 1). The two main ST complexes were ST10 (13 isolates; $14.3 \%$ ) and ST23 (10 isolates; $11 \%)$. Five of the eight most frequent STs, including ST131, were singletons (Table 1).

Analysis with eBURST provided an overview of STs of the Spanish ESBLproducing $E$. coli isolates from this study compared with all $E$. coli isolates from the MLST database. The major ST complexes found are represented in Fig. 1. ST10 complex was the most commonly detected in this study $(14.3 \%)$ and is also the most frequent ST in the MLST database (9.4\%).

\subsection{Sequence type distribution in relation to extended-spectrum $\beta$-lactamase} types

The Spanish ESBL-producing E. coli were distributed among a large number of MLST types (Table 1). The CTX-M-14-producers were distributed into 12 different ST complexes (28 isolates) and 10 singletons (13 isolates) (Table 1). 
The SHV-12-producers alone were distributed among six ST complexes (11 isolates) and 7 singletons (7 isolates) (Table 1). The CTX-M-9-producers alone were distributed among four ST complexes (8 isolates) and 7 singletons (9 isolates) (Table 1).

Escherichia coli ST131 isolates carried six different ESBLs: CTX-M-9; CTX-M10; CTX-M-1; CTX-M-14; CTX-M-15; and SHV-12. In addition, E. coli isolates belonging to the two major complexes, ST10 and ST23, had five and four ESBL types, respectively.

\subsection{Phylogenetic groups in relation to sequence types}

A strong association between STs and phylogenetic groups was found, as all 23 isolates of the ST10 and ST23 complexes were phylogroup A. Also, of eight ST131 isolates, seven belonged to the virulent extraintestinal phylogroup B2, and the three ST117 isolates belonged to phylogroup D (Table 1).

\section{Discussion}

We characterised the population structure of $92 \mathrm{E}$. coli isolates carrying eight types of ESBLs, seven of the CTX-M family. Several findings are of epidemiological and microbiological interest: (i) a great diversity of MLST types was observed; (ii) the E. coli isolates belonging to the most common STs or ST complexes could carry almost all of the ESBL types identified in this study; and

(iii) ST10 complex (the most frequent in this study and also in the MLST 
database) carried five different ESBLs (CTX-M-14, SHV-12, CTX-M-9, CTX-M15 and CTX-M-32).

The spread of ST131/B2 CTX-M-15-producing E. coli isolates, previously described in Madrid, Spain [5], and in other countries [6-8], was not observed in this study. In fact, of the three CTX-M-15-producing isolates, only one was ST131/B2 whilst the other two belonged to STs 167 and 653. Plasmids that carry CTX-M-15 are well disseminated among circulating virulent ST131/B2 E. coli strains in some geographic areas [6-8,13]; however, we found that ST131 E. coli not only carried CTX-M-15 but also CTX-M-9, CTX-M-10, CTX-M-14, SHV-12 and CTX-M-1. These results agree with the very recent description of ST131 E. coli harbouring blaCTX-M-2-group and bla $a_{\mathrm{CTX}-\mathrm{M}-14}$ in Japan [9]. Unlike the epidemic ST38 E. coli described in Japan that is associated with CTX-M-9group ESBLs [9], we detected only one ST38 isolate producing CTX-M-14.

In a recent French study, ST131 and ST10 complex isolates were found to be the most prevalent among faecal samples from healthy carriers of nalidixic acidresistant (but ESBL-negative) E. coli [14]. In England, 59\% of uropathogenic $E$. coli were ST131 (98.1\% of them resistant to cefpodoxime); the next most prevalent strains, ST73 and ST95 (6.8\% each) [15], were not detected in our study.

None of the ESBL-producing E. coli analysed in this study caused outbreaks in any of the participating Spanish hospitals. However, we have previously reported the epidemic dissemination of a clonal CTX-M-15-producing E. coli in 
community healthcare centres, long-term care facilities and hospitals in the Madrid area [5]. Two epidemiological scenarios are possible: the clonal spread of a few specific strains; and the dissemination of bla $a_{E S B L}$ genes among a diverse population of E. coli. The two models can occur simultaneously or separately, thereby contributing to the rapid dissemination of ESBLs.

In this study, we characterised the population structure of $92 \mathrm{E}$. coli isolates carrying eight ESBL types, with seven from the CTX-M family. Although a diversity of MLST types was observed, E. coli belonging to the most common STs or ST complexes (ST131, ST10 complex and ST23 complex) can carry almost all of the ESBL types. Escherichia coli ST131 carried not only CTX-M-15 but also CTX-M-9, CTX-M-10, CTX-M-14, SHV-12 and CTX-M-1.

Acknowledgments: The authors are grateful to the following participants for their help during collection of the isolates for this project: L. Matas and M. Giménez (Hospital Germans Trias i Pujol, Badalona); I. Sanfeliu (Corporació Hospitalària Parc Taulí, Sabadell); G. Prats (Hospital Vall d’Hebron, Barcelona); M. Ruíz and J. Vila (Hospital Clínic, Barcelona); A. Domínguez (Hospital de Bellvitge, Hospitalet); A. Guerrero and J. Colomina (Hospital de La Ribera, Alzira); A. Pascual (Hospital Virgen de la Macarena, Sevilla); and J. Aznar (Hospital Virgen del Rocío, Sevilla).

Funding: This study was partially supported by grants from the Fondo de Investigaciones Sanitarias (PI04339, PI040162, PI040837 and PI042012), the Instituto de Salud Carlos III - FEDER, Spanish Network for the Research in 
Infectious Diseases (REIPI C03/14) and the Spanish Network for the Research in Infectious Diseases (REIPI RD06/0008).

Competing interest: None declared.

Ethical approval: Not required. 


\section{References}

[1] Oteo J, Lázaro E, de Abajo FJ, Baquero F, Campos J. Antimicrobialresistant invasive Escherichia coli, Spain. Emerg Infect Dis 2005;11:546-53.

[2] Cantón R, Novais A, Valverde A, Machado E, Peixe L, Baquero F, et al. Prevalence and spread of extended-spectrum $\beta$-lactamase-producing Enterobacteriaceae in Europe. Clin Microbiol Infect 2008;14(Suppl 1):14453.

[3] García A, Navarro F, Miró E, Villa L, Mirelis B, Coll P, et al. Acquisition and diffusion of bla Microbiol Lett 2007;271:71-7.

[4] Novais A, Cantón R, Valverde A, Machado E, Galán JC, Peixe L, et al. Dissemination and persistence of bla containing CR1 associated with defective transposon derivatives from Tn402 located in early antibiotic resistance plasmids of IncHI2, IncP1-alpha, and IncFI groups. Antimicrob Agents Chemother 2006;50:2741-50.

[5] Oteo J, Navarro C, Cercenado E, Delgado-Iribarren A, Wilhelmi I, Orden B, et al. Spread of Escherichia coli strains with high-level cefotaxime and ceftazidime resistance between the community, long-term care facilities, and hospital institutions. J Clin Microbiol 2006;44:2359-66.

[6] Lau Siu Ha, Kaufmann ME, Livermore DM, Woodford N, Willshaw GA, Cheasty T, et al. UK epidemic Escherichia coli strains A-E, with CTX-M-15 $\beta$-lactamase, all belong to the international O25:H4-ST131 clone. J Antimicrob Chemother 2008;62:1241-4.

[7] Coque TM, Novais A, Carattoli A, Poirel L, Pitout J, Peixe L, et al. Dissemination of clonally related Escherichia coli strains expressing 
extended-spectrum $\beta$-lactamase CTX-M-15. Emerg Infect Dis 2008;14:195200.

[8] Nicolas-Chanoine MH, Blanco J, Leflon-Guibout V, Demarty R, Alonso MP, Caniça MM, et al. Intercontinental emergence of Escherichia coli clone O25:H4-ST131 producing CTX-M-15. J Antimicrob Chemother 2008;61:273-81.

[9] Suzuki S, Shibata N, Yamane K, Wachino J, Ito K, Arakawa Y. Change in the prevalence of extended-spectrum- $\beta$-lactamase-producing Escherichia coli in Japan by clonal spread. J Antimicrob Chemother 2009;63:72-9.

[10] Diestra K, Coque TM, Miró E, Oteo J, Nicolau CJ, Campos J, et al. Characterization and molecular epidemiology of ESBL in Escherichia coli and Klebsiella pneumoniae in 11 Spanish hospitals (2004) [in Spanish]. Enferm Infecc Microbiol Clin 2008;26:404-6.

[11] Diestra K, Juan C, Curiao T, Moyá B, Miró E, Oteo J, et al. Characterisation of plasmids encoding $b / a_{E S B L}$ and surrounding genes in Spanish clinical isolates of Escherichia coli and Klebsiella pneumoniae. J Antimicrob Chemother 2009;63:60-6.

[12] Clermont O, Bonacorsi S, Bingen E. Rapid and simple determination of the Escherichia coli phylogenetic group. Appl Environ Microbiol 2000;66:4555-8.

[13] Clermont O, Lavollay M, Vimont S, Deschamps C, Forestier C, Branger C, et al. The CTX-M-15-producing Escherichia coli diffusing clone belongs to a highly virulent B2 phylogenetic subgroup. J Antimicrob Chemother 2008;61:1024-8. 
[14] Leflon-Guibout V, Blanco J, Amaqdouf K, Mora A, Guize L, NicolasChanoine MH. Absence of CTX-M enzymes but a high prevalence of clones, including clone ST131, among the fecal Escherichia coli isolates of healthy subjects living in the Paris area. J Clin Microbiol 2008;46:3900-5.

[15] Lau SH, Reddy S, Cheesbrough J, Bolton FJ, Willshaw G, Cheasty T, et al. Major uropathogenic Escherichia coli strain isolated in the northwest of England identified by multilocus sequence typing. J Clin Microbiol 2008;46:1076-80. 
Fig. 1. Population snapshot of sequence types (STs) of all Escherichia coli isolates from the multilocus sequence typing (MLST) database and STs of extended-spectrum $\beta$-lactamse (ESBL)-producing $E$. coli from this study considered altogether. The most important ST complexes found in this study (ST10 complex, ST23 complex, ST31 complex, ST131, ST446 complex and ST538 complex) are emphasised. 
Table 1

Distribution of sequence type (ST) and phylogenetic groups among 91 extended-spectrum $\beta$-lactamse (ESBL)-producing Escherichia coli isolates in relation to ESBL type

$\mathrm{ESBL}(n) \quad \mathrm{ST}$ complex $\mathrm{ST}^{\mathrm{a}}$ (phylogenetic group)

$(n)$

CTX-M-14 ST23 (6) $23^{2}(\mathrm{~A}) ; 88^{1}(\mathrm{~A}) ; 410^{1}(\mathrm{~A}) ; 612^{1}(\mathrm{~A}) ; 650^{1}(\mathrm{~A})$

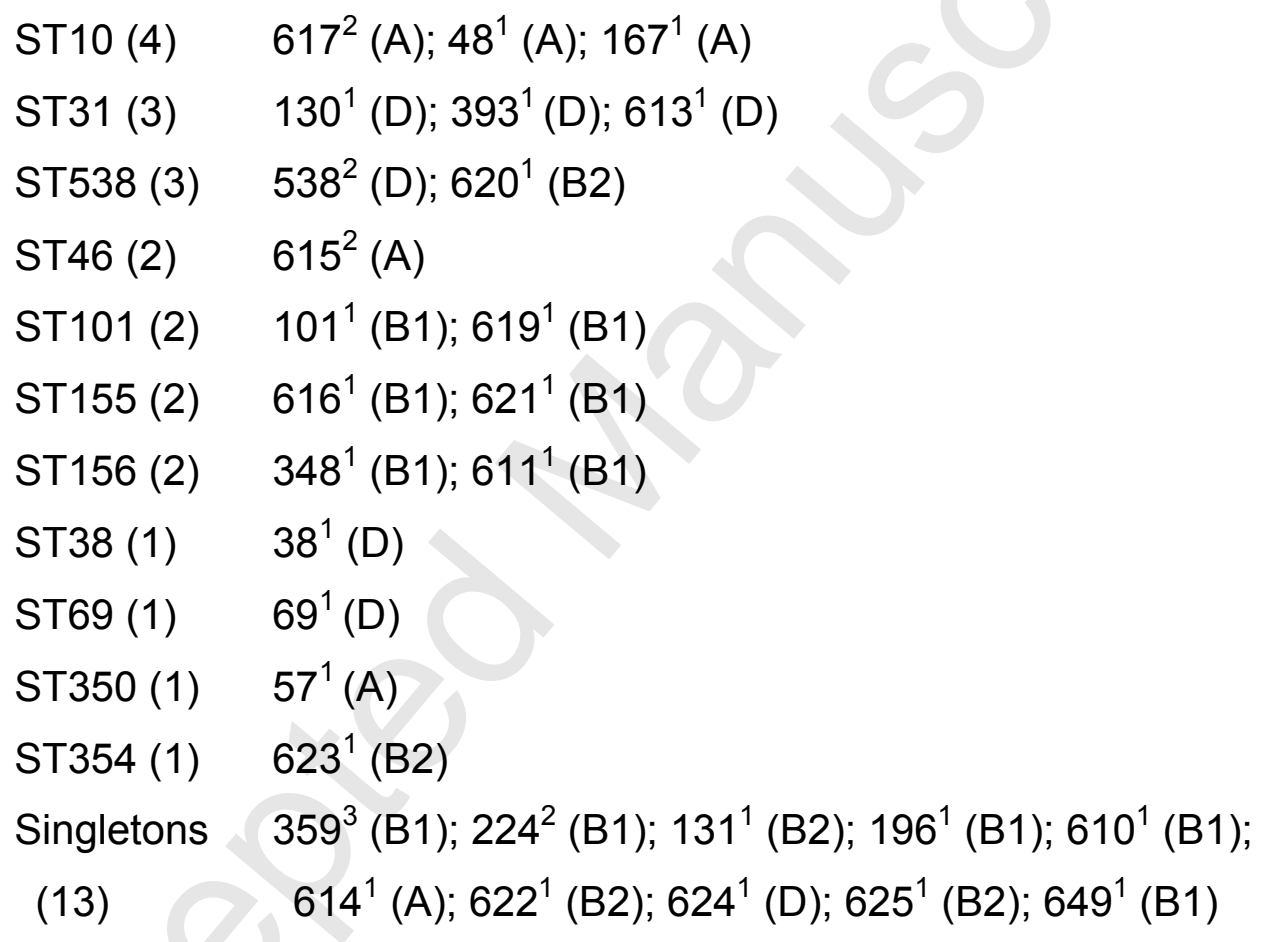

SHV-12 ST10 (4) $\quad 167^{2}(A) ; 10^{1}(A) ; 227^{1}(A)$

(18)

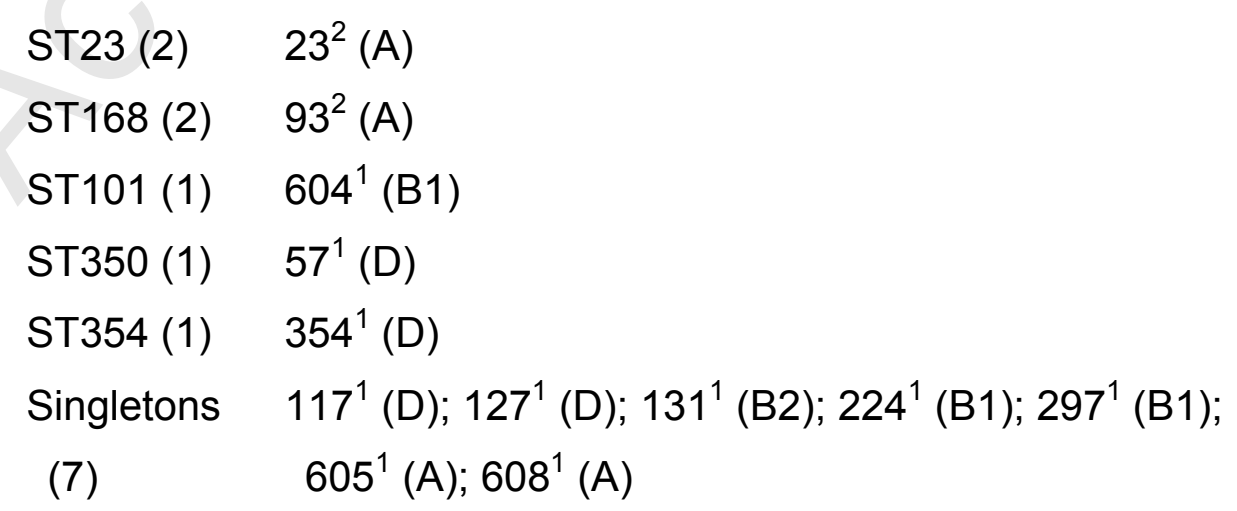

CTX-M-9 ST446 (4) $602^{4}$ (B1) 


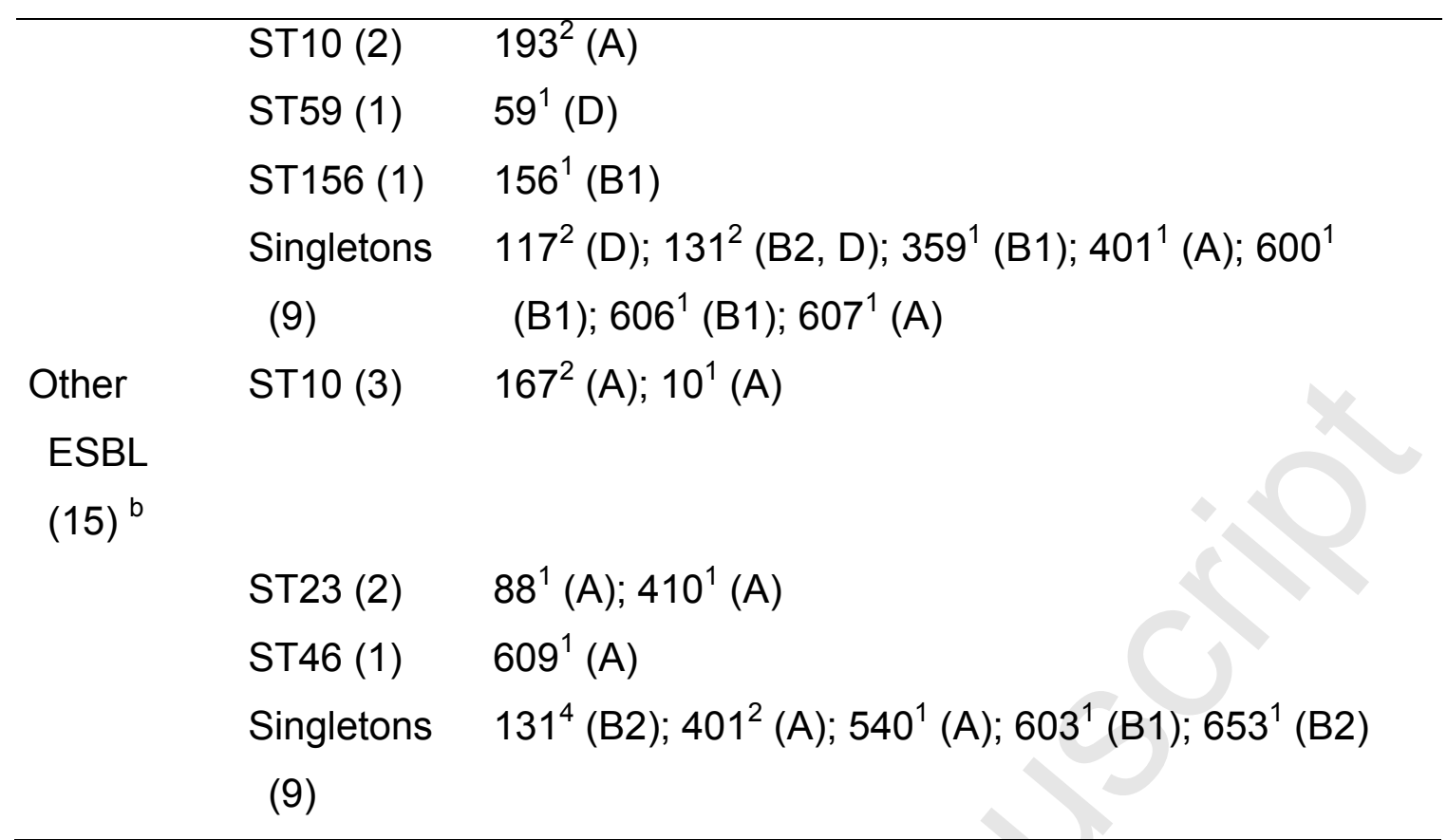

\footnotetext{
a Number of isolates in superscript.

b Two CTX-M-1, three CTX-M-3, two CTX-M-10, three CTX-M-15, three CTX-M32 and two CTX-M-9 + SHV-12.
} 


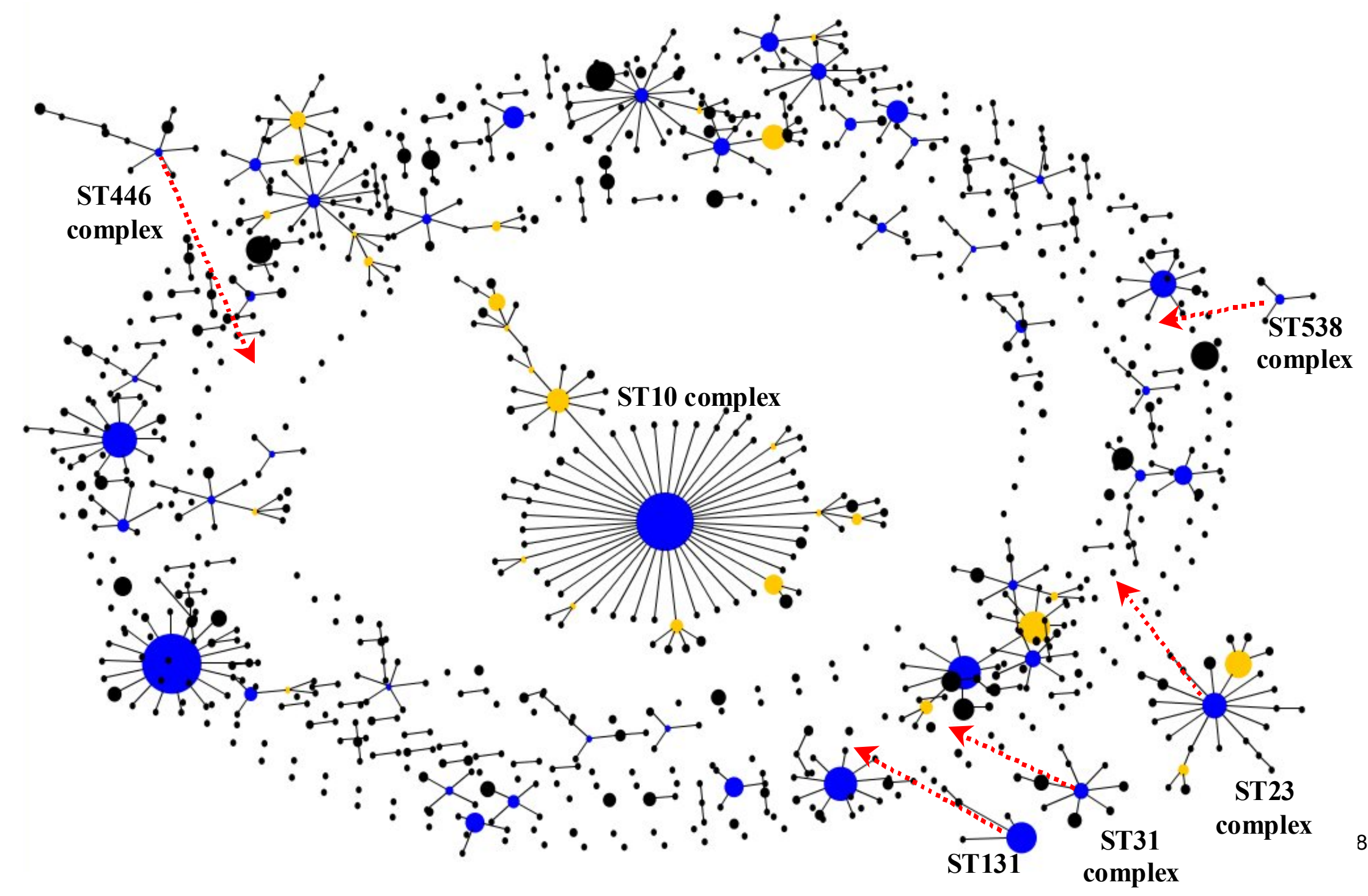

